\title{
Fuzzy attribute logic with model constraints
}

\author{
Radim Belohlavek $^{1}$ Vilem Vychodil ${ }^{1}$ \\ ${ }^{1}$ Dept. Computer Science, Palacky University, Olomouc, Czech Republic
}

\begin{abstract}
Presented are preliminary results on ordinary-style and graded-style completeness results for fuzzy attribute logic with models constrained by fuzzy closure operators with hedges.
\end{abstract}

Keywords: Attribute implication, entailment, axiomatization, background knowledge, constraint

\section{Introduction and problem setting}

This paper deals with rules of the form $E \Rightarrow F$, where $E$ and $F$ can be seen as conjunctions of attributes, i.e., names of properties that an object can have or a collection of object can share. Formulas of this type are widely used in computer science, e.g., in data mining as attribute implications [13] and association rules or in database systems as functional dependencies [20]. In the data-mining sense, $E \Rightarrow F$ is interpreted as a rule saying: "if an object has all the attributes from $E$, then it has all the attributes from $F$ ". As functional dependency, $E \Rightarrow F$ is interpreted as "if two tuples (or database table) have the same values on all the attributes from $E$, then they have the same values on all the attributes from $F$ ". Although these two interpretations are different, the notions of semantic entailments based on the different interpretations are the same, see [20]. In addition, the semantic entailment is axiomatizable using a small set of deduction rules, called Armstrong rules [1].

The objective of this paper is to present a preliminary study of entailment of analogous rules from the perspective of fuzzy logic considering, in addition, constraints on permissible models. The motivation for a graded extension stems from the fact that " $x$ having an attribute $y$ " is, in many cases, a graded relationship rather than bivalent one. Thus, we may wish to consider rules saying, e.g., "if an athlete is explosive to degree $a$ and lightweight to degree $b$, then he will be fast on short distances to degree $c$ " which can be seen as a generalized attribute implications where attributes are permitted to be weighted by truth degrees. A different motivation can be found in database systems with domains equipped with similarity relations $[7,10]$ cf. also [11]. Then, it makes sense to consider rules like "if two athletes have similar blood pressure to degree $a$ and similar speed on short distances to degree $b$, then they have similar speed on longer distances to degree $c$ ". An important feature that such rules can be considered true to various degrees (fully true, more or less true, not at all true,... ). This paper develops a complete logic, called fuzzy attribute logic, for dealing with such rules.

Model constraints can be seen as constraints on permissible models. Instead of using all possible models, we restrict ourselves to models that are fixed points of a particular closure operator. The reason is that when reasoning with the abovementioned rules, one is often interested only in rules, where the antecedent (part before $\Rightarrow$ ) and consequent (part after $\Rightarrow$ ) satisfies an additional condition. In addition, such rules are evaluated in models that also satisfy the condition. We showed in [8], many natural constraints can be formulated by means of fixed points of closure operators. For instance, constraints by the size of support, which are crucial in mining association rules, are a particular example of this constraint. Therefore, we present here an extension of fuzzy attribute logic considering additional model constraints.

The paper is organized as follows. Section 2 contains preliminaries, Section 3 introduces formulas, constraints, and semantic entailment. In Section 4 , we introduce the deductive system and show its soundness. Section 5 presents various completeness results (ordinary-style completeness and graded-style completeness).

\section{Preliminaries}

The logic of fuzzy attribute implications with model constraints will be developed over complete residuated lattices with truth-stressing hedges (shortly, hedges). Recall that a complete residuated lattice with a hedge is an algebra $\mathbf{L}=\langle L, \wedge, \vee, \otimes, \rightarrow$ $\left.,{ }^{*}, 0,1\right\rangle$, where $\langle L, \wedge, \vee, 0,1\rangle$ is a complete lattice, $\langle L, \otimes, 1\rangle$ is a commutative monoid, and $\otimes$ and $\rightarrow$ satisfy the adjointness property: $a \otimes b \leq c$ iff $a \leq b \rightarrow c$ for any $a, b, c \in L$. Hedge ${ }^{*}$ is a unary operation $^{*}: L \rightarrow L$ satisfying

$$
\begin{aligned}
1^{*} & =1, \\
a^{*} & \leq a, \\
(a \rightarrow b)^{*} & \leq a^{*} \rightarrow b^{*}, \\
a^{* *} & =a^{*},
\end{aligned}
$$

for all $a, b \in L$. As usual, $\otimes$ and $\rightarrow$ serve as truth functions of binary logical connectives "fuzzy conjunction" and "fuzzy implication". The mutual relationship of $\otimes$ and $\rightarrow$ posed by adjointness stems from a graded generalization of modus ponens, see $[3,15,17]$. The hedge ${ }^{*}$ serves as 
a truth function of logical connective "very true", see $[17,18]$. Properties (1)-(4) have natural interpretations, e.g. (3) can be read: "if $a \rightarrow b$ is very true and if $a$ is very true, then $b$ is very true", etc.

Examples of (complete) residuated lattices include residuated lattices on the real unit interval given by left-continuous t-norms $[12,19]$ and can be found in monographs $[3,17]$. Two boundary cases of hedges are (i) identity, i.e. $a^{*}=a(a \in L)$; (ii) globalization [23] which can be seen as a truth function of connective "fully true": $1^{*}=1$ and $a^{*}=0$ if $a<1$.

Remark 1. Note that globalization agrees with Baaz's $\Delta$-operation [2] in case of linearly ordered residuated lattices. Also note that the conditions (1)-(4) posed on hedges are slightly different from those presented in [18]. Namely, we do not require $(a \vee b)^{*} \leq a^{*} \vee b^{*}$ which is used in [18]. On the other hand, we use (4) which is not used in [18]. As we will see later, we introduce (4) for technical reasons. In practice, a hedge * satisfying (1)-(4) is an interior operator.

We now recall basic notions of fuzzy relational structures that will be used throughout the paper. Given a complete residuated lattice $\mathbf{L}$, we define usual notions: an L-set (fuzzy set) $A$ in universe $U$ is a map $A: U \rightarrow L, A(u)$ being interpreted as "the degree to which $u$ belongs to $A$ ". $L^{U}$ denotes the collection of all $\mathbf{L}$-sets in $U$. The operations with $\mathbf{L}$-sets are defined componentwise. For instance, intersection of $\mathbf{L}$-sets $A, B \in L^{U}$ is an $\mathbf{L}$ set $A \cap B$ in $U$ such that $(A \cap B)(u)=A(u) \wedge B(u)$ for each $u \in U$, etc. For $a \in L$ and $A \in \mathbf{L}^{U}$, we define L-sets $a \otimes A$ ( $a$-multiple of $A$ ) and $a \rightarrow A$ $(a$-shift of $A)$ by $(a \otimes A)(u)=a \otimes A(u),(a \rightarrow$ $A)(u)=a \rightarrow A(u)(u \in U)$. Given $A, B \in L^{U}$, we define a subsethood degree of $A$ in $B$ as follows: $S(A, B)=\bigwedge_{u \in U}(A(u) \rightarrow B(u))$. Described verbally, $S(A, B)$ represents the degree to which $A$ is a subset of $B$. In addition, we write $A \subseteq B$ iff $S(A, B)=1$, i.e. if $A$ is fully included in $B$. Using adjointness, we get that $A \subseteq B$ iff, for each $u \in U$, $A(u) \leq B(u)$.

Recall from [4] that a system $\mathcal{S} \subseteq L^{U}$ of fuzzy sets is called an $\mathbf{L}^{*}$-closure system on $U$ if (i), for any fuzzy sets $A_{i} \in \mathcal{S}(i \in I)$ we have $\bigcap_{i \in I} A_{i} \in \mathcal{S}$, and (ii) for any fuzzy set $A \in \mathcal{S}$ and a truth degree $a \in L, a^{*} \rightarrow A \in \mathcal{S}$. Thus, $\mathcal{S}$ is an $\mathbf{L}^{*}$-closure system if it is closed under arbitrary intersections and $a^{*}$ shifts. An $\mathbf{L}^{*}$-closure operator on $U$ is any map $C: L^{U} \rightarrow L^{U}$ satisfying, for each $A, B_{1}, B_{2} \in L^{U}$,

$$
\begin{aligned}
A & \subseteq C(A), \\
S\left(B_{1}, B_{2}\right)^{*} & \leq S\left(C\left(B_{1}\right), C\left(B_{2}\right)\right), \\
C(A) & =C(C(A)) .
\end{aligned}
$$

Observe that (6) can be read: "if $B_{1}$ is very included in $B_{2}$, then $C\left(B_{1}\right)$ is included in $C\left(B_{2}\right)$ ". Put in words, ${ }^{*}$ which appears in (6) plays the role of a parameter of monotony. With stronger hedges, we put more emphasis on $B_{1}$ being a subset of $B_{2}$. $\mathbf{L}^{*}$-closure systems and operators have a mutual relationship which is analogous to ordinary closure systems and closure operators. Namely, (i) a set of all fixed points of an $\mathbf{L}^{*}$-closure operator forms an $\mathbf{L}^{*}$-closure system; (ii) a map assigning to each L-set $A \in L^{U}$ the least element of an $\mathbf{L}^{*}$-closure system which contains $A$ is an $\mathbf{L}^{*}$-closure operator; (iii) there is a one-to-one correspondence between $\mathbf{L}^{*}$-closure systems and $\mathbf{L}^{*}$-closure operators, see [4] for details. In the sequel, we will denote by $\operatorname{fix}(C)$ the set of all fixed points of an $\mathbf{L}^{*}$-closure operator $C: L^{U} \rightarrow L^{U}$, i.e.

$$
\begin{aligned}
\operatorname{fix}(C) & =\left\{A \in L^{U} \mid A=C(A)\right\} \\
& =\left\{C(A) \mid A \in L^{U}\right\} .
\end{aligned}
$$

In the following we use well-known properties of residuated lattices and fuzzy structures which can be found, e.g., in monographs $[3,17]$.

\section{Constrained fuzzy attribute implications}

This section introduces formulas of our logic, so called fuzzy attribute $C$-implications, constraints on models, and notion of semantic entailment.

\subsection{Definition and interpretation}

Fuzzy attribute implications and their semantics have been introduced and studied in $[5,6,9]$. Recall that given a finite set $Y$, a fuzzy attribute implication (over attributes $Y$ ), we mean an expression $E \Rightarrow F$, where $E, F \in L^{Y}$ (i.e., $A$ and $B$ are fuzzy sets of attributes), and $L$ is a support of a complete residuated lattice $\mathbf{L}$.

Remark 2. Each $y \in Y$ is called an attribute. Attributes can be understood as names of properties/features which may be shared by objects. Since we are interested in graded extension ordinary attribute implications, we allow object to have attribute to degrees (taken from $L$ ).

In order to consider truth of fuzzy attribute implications, we introduce a semantic component (a structure) in which we evaluate the formulas and their formal interpretation. The informal meaning of $E \Rightarrow F$ is: "if it is (very) true that an object has all attributes from $E$, then it has also all attributes from $F$ ". Formally, for an $\mathbf{L}$-set $M \in L^{Y}$ of attributes, we define a degree $\|E \Rightarrow F\|_{M} \in L$ to which $E \Rightarrow F$ is true in $M$ by

$$
\|E \Rightarrow F\|_{M}=S(E, M)^{*} \rightarrow S(F, M),
$$

where $S(\cdots)$ denote subsethood degrees, and $\rightarrow$ and * are operations of a "fuzzy conjunction" and a hedge of a complete residuated lattice $\mathbf{L}$, see Section 2 .

Remark 3. The degree $\|E \Rightarrow F\|_{M}$ can be understood as follows: if $M$ (semantic component) represents presence of attributes of some object, i.e. 
$M(y)$ is truth degree to which "the object has the attribute $y \in Y$ ", then $\|E \Rightarrow F\|_{M}$ is the truth degree of "if the object has all attributes from $E$, then it has all attributes from $F$ ", which corresponds to the desired interpretation of $E \Rightarrow F$.

Note that fuzzy attribute implications can be represented as formulas of first-order predicate fuzzy logics (in narrow sense) and their interpretation can be expressed as truth of the formulas in fuzzy structures [3, 17]. Indeed, one can consider a first-order language with unary relation symbols $e, f, m$ and pairwise different symbols for constants $y_{1}, \ldots, y_{n}$ (nullary function symbols). Then a fuzzy attribute implication can be seen as a formula of the form

$$
\begin{aligned}
& \Delta\left(\left(e\left(y_{1}\right) \Rightarrow m\left(y_{1}\right)\right) \sqcap \cdots \sqcap\left(e\left(y_{n}\right) \Rightarrow m\left(y_{n}\right)\right)\right) \\
& \Rightarrow\left(\left(f\left(y_{1}\right) \Rightarrow m\left(y_{1}\right)\right) \sqcap \cdots \sqcap\left(f\left(y_{n}\right) \Rightarrow m\left(y_{n}\right)\right)\right),
\end{aligned}
$$

where $\Pi$ is a symbol for min-conjunction (i.e., a conjunction interpreted by $\wedge$ ), $\Rightarrow$ is a symbol for implication (interpreted by $\rightarrow$ ), and $\Delta$ is a symbol for unary connective (interpreted by ${ }^{*}$ ). Denote such a formula by $\varphi$.

Observe that using the standard interpretation of $\varphi$ in a first-order fuzzy structure $\mathbf{M}$ where each $y_{i}$ is interpreted by a constant $y_{i}^{\mathbf{M}}$ from its universe $M$ and any of $e, f$, and $m$ is interpreted by an $\mathbf{L}$-set $e^{\mathbf{M}}, f^{\mathbf{M}}$, and $m^{\mathbf{M}}$ in $M$, respectively, $\varphi$ can be read: "if it is very true that $y_{1}$ is in $m_{1}$ at least to degree to which $y_{1}$ is in $e_{1}$ and, .., and $y_{n}$ is in $m_{n}$ at least to degree to which $y_{n}$ is in $e_{n}$, then $y_{1}$ is in $m_{1}$ at least to degree to which $y_{1}$ is in $f_{1}$ and,..., and $y_{n}$ is in $m_{n}$ at least to degree to which $y_{n}$ is in $f_{n}$ ".

Hence, for $\varphi$, we can consider L-sets $M\left(y_{i}\right)=$ $m^{\mathbf{M}}\left(y_{i}^{\mathbf{M}}\right), E\left(y_{i}\right)=e^{\mathbf{M}}\left(y_{i}^{\mathbf{M}}\right), F\left(y_{i}\right)=f^{\mathbf{M}}\left(y_{i}^{\mathbf{M}}\right)$. Under this notation, we have $\|\varphi\|_{\mathbf{M}}=\|E \Rightarrow F\|_{M}$, where $\|\varphi\|_{\mathbf{M}}$ is the degree to which $\varphi$ is true in $\mathbf{M}$ as it is usually defined, see $[3,17]$ for details.

Hence, $E \Rightarrow F$ can be seen a abbreviation for $\varphi$. Working with such an abbreviation instead of $\varphi$ is beneficial mainly for technical reasons (the representation is concise).

Remark 4. Note that the hedge * serves as a modifier of interpretation of $E \Rightarrow F$, see [6] for details. For instance, if ${ }^{*}$ is globalization then $\|E \Rightarrow F\|_{M}=$ 1 (i.e. $E \Rightarrow F$ is true in $M$ ) means that if $E \subseteq M$ then $F \subseteq M$, i.e. only full subsethood is taken into account. If * is identity then $\|E \Rightarrow F\|_{M}=1$ means $S(E, M) \leq S(F, M)$, i.e. any subsethood degree is taken into account.

\subsection{Constraints by $\mathrm{L}^{*}$-closure operators}

The definitions of fuzzy attribute implications and models use arbitrary L-sets in $Y$. For an expert, who uses such formulas, e.g., to describe dependencies in a problem domain, many $\mathbf{L}$-sets in $Y$ do not represent "interesting collections of attributes". The expert may wish to restrict himself only to a particular subset $\mathcal{S} \subseteq L^{Y}$. Then, only $E \Rightarrow F$ where
$E, F \in \mathcal{S}$ will be taken into consideration. Similarly, degrees $\|\cdots\|_{M}$ will be considered only if $M \in \mathcal{S}$.

In this paper, we assume that $\mathcal{S}$ is an $\mathbf{L}^{*}$ closure system. Because of the correspondence of $\mathbf{L}^{*}$-closure systems and $\mathbf{L}^{*}$-closure operators, $\mathcal{S}=$ fix $(C)$ for some $\mathbf{L}^{*}$-closure operator $C: L^{Y} \rightarrow L^{Y}$. The operator can be seen so that given $M \in L^{Y}$, the value of $C(M)$ is the least "interesting" (i.e., constrained) $\mathbf{L}$-set in $L^{Y}$ according to the constraint represented by $C$.

Given the set of attributes $Y$ and $C: L^{Y} \rightarrow L^{Y}$, a fuzzy attribute implication $E \Rightarrow F$ such that $C(E)=E$ and $C(F)=F$ shall be called a fuzzy attribute implication constrained by $C$ (shortly, a fuzzy attribute $C$-implication).

Remark 5. (1) Note that the requirement for $C$ being an $\mathbf{L}^{*}$-closure operator is a technical one (with this requirement, the notions of semantic entailment and provability introduced hereinafter enjoy fruitful properties). On the other hand, many natural constraints can be represented by $\mathbf{L}^{*}$-closure operators, see [8] for examples.

(2) As a special case, for $C: L^{Y} \rightarrow L^{Y}$ being the identity map $\left(C(E)=E\right.$ for each $\left.E \in L^{Y}\right)$, fuzzy attribute $C$-implications are the ordinary (unconstrained) fuzzy attribute implications.

In the next section, we introduce semantic entailment of fuzzy attribute $C$-implications.

\subsection{Semantic entailment}

Let $C: L^{Y} \rightarrow L^{Y}$ is an $\mathbf{L}^{*}$-closure operator describing a constraint. Let $T$ be a set of fuzzy attribute $C$-implications. A fixed point $M \in \operatorname{fix}(C)$ is called a $C$-model of $T$ if $\|E \Rightarrow F\|_{M}=1$ for each $E \Rightarrow F \in T$. The set of all $C$-models of $T$ shall be denoted by $\operatorname{Mod}_{C}(T)$, i.e.

$$
\operatorname{Mod}_{C}(T)=\{M \in \operatorname{fix}(C) \mid M \text { is } C \text {-model of } T\} .
$$

A degree $\|E \Rightarrow F\|_{T}^{C} \in L$ to which a fuzzy attribute $C$-implication $E \Rightarrow F$ semantically follows from $T$ is defined by

$$
\|E \Rightarrow F\|_{T}^{C}=\bigwedge_{M \in \operatorname{Mod}_{C}(T)}\|E \Rightarrow F\|_{M} .
$$

Described verbally, $\|E \Rightarrow F\|_{T}^{C}$ is defined as a degree to which " $E \Rightarrow F$ is true in each $C$-model of $T$ ". Hence, $\|\cdots\|_{T}^{C}$ defined by (10) represents a degree of semantic entailment from $T$ under $C$.

If $C$ is the identity (no constraint), then $\| E \Rightarrow$ $F \|\left.\right|_{T} ^{C}$ becomes $\|E \Rightarrow F\|_{T}$ as it is defined in [6].

Remark 6 . The database interpretation of fuzzy attribute implication mentioned in Section 1 will not be described in this paper since both of the interpretations have the same concept of semantic entailment. That is a degree $\|E \Rightarrow F\|_{T}^{C}$ defined by (10) can be shown to be exactly the degree which $E \Rightarrow F$ semantically follows from $T$ under $C$ when using the database interpretation. Therefore, a logical calculus which is complete (either in ordinary 
or in graded style) with respect to one of these interpretations is also complete with respect to the other interpretation. For details we refer to [7].

Recall that the (unconstrained) fuzzy attribute $\operatorname{logic}$ (when $C$ is identity) is both ordinary-style and graded-style complete, see [9]. We can obtain the notion of provability from [9] to a completeness results for our logic with any $C$. Indeed, the ordinary provability $\vdash$ of fuzzy attribute implications from a $C$-theory can be used to characterize semantic entailment from $T$ under $C$ provided that we consider provability form $T$ enriched by additional fuzzy attribute implications. Indeed, for any $C$-theory $T$, we can define

$$
T_{C}=T \cup \operatorname{Th}(C),
$$

where $\operatorname{Th}(C)$ satisfies $\operatorname{Mod}(\operatorname{Th}(C))=\operatorname{fix}(C)$. Such $\operatorname{Th}(C)$ always exists. For instance, for

$$
\operatorname{Th}(C)=\left\{E \Rightarrow C(E) \mid E \in L^{Y}\right\},
$$

we get that the models of $\operatorname{Th}(C)$ are exactly the fixed points of $C$. Using $T_{C}$, we can get the following characterization:

Theorem 1. For any $C$-theory $T$ any fuzzy attribute $C$-implication $E \Rightarrow F$, we have

$$
T_{C} \vdash E \Rightarrow F \quad \text { iff } \quad\|E \Rightarrow F\|_{T}^{C}=1,
$$

where $T_{C}$ is given by (11) with $\operatorname{Th}(C)$ being (12).

Theorem 1 can be seen as a completeness result for entailment under $C$ that uses the ordinary notion of provability of fuzzy attribute implications. Therefore, in general, proofs of fuzzy attribute $C$ implications from $T_{C}$ contain unconstrained formulas. This kind of mixing of constrained and unconstrained formulas in proofs may be seen as an undesirable feature. Therefore, we might be interested in finding a deductive system that works solely with constrained formulas and related notion of provability. This will be the subject of further sections.

\section{Deduction rules and soundness}

In this section, we introduce a deductive system for fuzzy attribute logic with model constraints and prove its soundness over all $C$-models. We begin with deduction rules and appropriate notions of proofs and provability.

Our deductive system will consist of three independent deduction rules:

\footnotetext{
$\left(\mathrm{Ax}_{C}\right)$ infer $C(E \cup F) \Rightarrow F$, where $F \in \operatorname{fix}(C)$,

$\left(\mathrm{Cut}_{C}\right)$ from $E \Rightarrow F$ and $C(F \cup G) \Rightarrow H$ such that $E, F, H \in \operatorname{fix}(C)$ and $G \in L^{Y}$ infer $C(E \cup G) \Rightarrow H$,

$\left(\mathrm{Mul}_{C}\right)$ from $E \Rightarrow F$ such that $E, F \in \operatorname{fix}(C)$ infer $a^{*} \otimes E \Rightarrow a^{*} \otimes F$ for any $a \in L$.
}

Remark 7. The rules are inspired by Armstrong-like axioms which are well known in database systems, see [1] and also [20] for a good overview. Also note that $\left(\mathrm{Ax}_{C}\right)$ is, in fact, an axiom scheme stating that each $C(E \cup F) \Rightarrow F$ is derivable in a single step (from no preceding formulas). On the other hand, $\left(\mathrm{Cut}_{C}\right)$ is a deduction rule which, considering two formulas of the form $E \Rightarrow F$ and $C(F \cup G) \Rightarrow H$ as the input (the part of the rules preceding "infer"), produces a single formula $C(E \cup G) \Rightarrow H$ as the output (the part of the rule following "infer"). Analogously, $\left(\mathrm{Mul}_{C}\right)$ can be used to produce $a^{*} \otimes E \Rightarrow a^{*} \otimes F$ given $E \Rightarrow F$.

Using $\left(\mathrm{Ax}_{C}\right)-\left(\mathrm{Cut}_{C}\right)$, we define the notions of a proof and a provability. A sequence $\varphi_{1}, \ldots, \varphi_{n}$ of fuzzy attribute $C$-implications is called a $C$-proof of $\varphi$ from $T$ if $\varphi_{n}=\varphi$ and, for each $i=1, \ldots, n$, one of the following conditions holds

(i) $\varphi_{i} \in T$,

(ii) $\varphi_{i}$ results using $\left(\mathrm{Ax}_{C}\right)$,

(iii) $\varphi_{i}$ results from $\varphi_{j}, \varphi_{k}(j, k<i)$ using $\left(\operatorname{Cut}_{C}\right)$,

(iv) $\varphi_{i}$ results from $\varphi_{j}(j<i)$ using $\left(\operatorname{Mul}_{C}\right)$.

A fuzzy attribute $C$-implication $E \Rightarrow F$ is $C$ provable from $T$, written $T \vdash_{C} E \Rightarrow F$, if there is a $C$-proof of $E \Rightarrow F$ from $T$. If $C$ is clear from the context, we say just "proof" and "provable" instead of " $C$-proof" and " $C$-provable", respectively.

Remark 8. If $C: L^{Y} \rightarrow L^{Y}$ is the identity map, then $\left(\mathrm{Ax}_{C}\right)-\left(\mathrm{Mul}_{C}\right)$ become the rules of (unconstrained) fuzzy attribute logic, cf. [9]. In that case, the notions of $C$-proofs and $C$-provability become the notions of proofs and provability as in [9]. This is how the present approach generalizes the observations from [9].

The following assertion shows basic properties of $\left(\mathrm{Ax}_{C}\right)-\left(\mathrm{Mul}_{C}\right)$.

Lemma 1. If $F, M \in \operatorname{fix}(C)$ and $E \in L^{Y}$, then $\|C(E \cup F) \Rightarrow F\|_{M}=1$.

If $\|E \Rightarrow F\|_{M}=1$ and $\|C(F \cup G) \Rightarrow H\|_{M}=1$ for $E, F, H, M \in \operatorname{fix}(C)$ and $G \in L^{Y}$, then $\| C(E \cup$ $G) \Rightarrow H \|_{M}=1$.

If $\|E \Rightarrow F\|_{M}=1$ for $E, F, M \in \operatorname{fix}(C)$, then $\left\|a^{*} \otimes E \Rightarrow a^{*} \otimes F\right\|_{M}=1$ for any $a \in L$.

The deductive system presented in this section is sound in the following sense:

Theorem 2 (soundless). Each of the rules $\left(\mathrm{Ax}_{C}\right)$, $\left(\mathrm{Cut}_{C}\right)$, and $\left(\mathrm{Mul}_{C}\right)$ is sound over all $C$-models. That is, if $T \vdash_{C} A \Rightarrow B$ then $\|A \Rightarrow B\|_{T}^{C}=1$.

\section{Completeness}

This section deals with completeness issues. We begin with showing ordinary-style completeness of $\left(\mathrm{Ax}_{C}\right)-\left(\mathrm{Mul}_{C}\right)$ over $C$-models, meaning that a fuzzy attribute $C$-implication is provable from a theory $T$ iff it semantically follows from $T$ to degree 1 . 
First, we prove the result for over all finite residuated lattices taken as structures of truth degrees. Second, we introduce an additional infinitary deduction rule which will allow us to attain ordinary-style completeness over all complete residuated lattices. Later on, we introduce degrees of provability and prove a graded-style (Pavelka-style [21]) completeness. We conclude the section by extending the previous results by considering theories as fuzzy sets of formulas.

\subsection{Ordinary-style completeness}

In this section, we establish ordinary-style completeness over any finite residuated lattice. Thus, throughout the section we assume that $\mathbf{L}$ is finite. We begin with technical observations on properties of $C$-provability and $C$-models that will be used to prove completeness.

Lemma 2. Let $T$ be a $C$-theory and $E \in \operatorname{fix}(C)$. Then, a system $\mathcal{A}_{T}^{C}(E) \subseteq \operatorname{fix}(C)$ of $\mathbf{L}$-sets given by

$$
\mathcal{A}_{T}^{C}(E)=\left\{F \in \operatorname{fix}(C) \mid T \vdash_{C} E \Rightarrow F\right\}
$$

has a greatest element, i.e., $\bigcup \mathcal{A}_{T}^{C}(E) \in \mathcal{A}_{T}^{C}(E)$. Moreover, $\bigcup \mathcal{A}_{T}^{C}(E) \in \operatorname{Mod}_{C}(T)$.

Lemma 3. Let $T$ be a $C$-theory and $E, F \in \operatorname{fix}(C)$ such that $T \nvdash_{C} E \Rightarrow F$. Then $\|E \Rightarrow F\|_{M}<1$ for some $M \in \operatorname{Mod}_{C}(T)$.

Using Lemma 3 and Theorem 2, we come up with the following result.

Theorem 3 (ordinary-style completeness). Let $\mathbf{L}$ be finite, $T$ be a $C$-theory. Then, for any fuzzy attribute $C$-implication $E \Rightarrow F$,

$$
T \vdash_{C} E \Rightarrow F \quad \text { iff } \quad\|E \Rightarrow F\|_{T}^{C}=1 .
$$

\subsection{Dealing with infinite $\mathbf{L}$}

Theorem 3 is confined to finite residuated lattices. It can be easily shown that $\left(\mathrm{Ax}_{C}\right)-\left(\mathrm{Cut}_{C}\right)$ are not complete over $C$-models considering arbitrary complete residuated lattice (including infinite ones). On the other hand, we can take an approach described in [9] and extend the deductive system by an additional infinitary deduction rule:

$\left(\operatorname{Add}_{C}^{\omega}\right)$ from $E \Rightarrow F_{i}(i \in I)$

$$
\text { such that } E, F_{i} \in \operatorname{fix}(C)(i \in I)
$$$$
\text { infer } E \Rightarrow C\left(\bigcup_{i \in I} F_{i}\right) \text {. }
$$

Notice that $\left(\operatorname{Add}_{C}^{\omega}\right)$ contains infinitely many formulas in the input part (the part preceding "infer"). Infinitary rules like $\left(\operatorname{Add}_{C}^{\omega}\right)$ are widely used in logic and computer science. For instance, they are used in universal algebra [24] as well as fuzzy logic in narrow sense (e.g., in TT $\forall$, see [17]).

In order to use $\left(\operatorname{Add}_{C}^{\omega}\right)$, we have to generalize the notion of a proof and consider proofs as certain labeled infinitely branching rooted (directed) trees with finite depth $[16,24]$ : under the notation $\mathcal{T}=$ $\langle l, S t\rangle$ which means that $\mathcal{T}$ is a tree with root label $l$ (a formula) and subtrees from a set $S t$, we can define proofs as follows.

Given a set $T$ of fuzzy attribute $C$-implications, a $C$-proof from $T$ is defined by two rules:

(i) for each $E \Rightarrow F \in T$, tuple $\mathcal{T}=\langle E \Rightarrow F, \emptyset\rangle$ is a $C$-proof from $T$,

(ii) if $\mathcal{T}_{i}=\left\langle\varphi_{i}, \ldots\right\rangle(i \in I)$ are $C$-proofs from $T$ and if $\varphi$ results from $\varphi_{i}(i \in I)$ by any of the deduction rules $\left(\mathrm{Ax}_{C}\right),\left(\mathrm{Cut}_{C}\right),\left(\mathrm{Mul}_{C}\right)$, or $\left(\operatorname{Add}_{C}^{\omega}\right)$, then $\mathcal{T}=\left\langle\varphi,\left\{\mathcal{T}_{i} \mid i \in I\right\}\right\rangle$ is a $C$-proof from $T$.

The generalized notion of $C$-proof gives a generalized notion of $C$-provability: $E \Rightarrow F$ is $C$-provable from $T$, written $T \vdash_{C}^{\omega} E \Rightarrow F$, if there is a $C$-proof (in the generalized sense) of $E \Rightarrow F$ from $T$.

We can show that the generalized notion of a $C$ proof is sufficient to characterize semantic entailment using arbitrary complete residuated lattice:

Theorem 4. Let $\mathbf{L}$ be a complete residuated lattice with hedge and $T$ be a $C$-theory. Then, for any fuzzy attribute $C$-implication $E \Rightarrow F$,

$$
T \vdash_{C}^{\omega} E \Rightarrow F \quad \text { iff } \quad\|E \Rightarrow F\|_{T}^{C}=1 .
$$

\subsection{Graded-style completeness}

Since semantic entailment is a graded notion and we work with a fixed (but arbitrary) complete residuated lattice, it might be tempting to provide a full characterization of $\|E \Rightarrow F\|_{T}^{C}$ by means of provability degrees. In this section, we show that in case of fuzzy attribute logic with model constraints, such characterization is possible.

We take a reductionist approach to the problem: we introduce provability degrees which are expressible by the ordinary-style provability and show that degrees $\|\cdots\|_{T}^{C}$ of semantic entailment can be expressed via formulas which are entailed to degree 1 (fully entailed). After that, we will be able to utilize the ordinary-style completeness results to obtain graded completeness.

For any $C$-theory $T$, define a degree $|E \Rightarrow F|_{T}^{C}$ to which a fuzzy attribute $C$-implication $E \Rightarrow F$ is provable from $T$ by

$$
|E \Rightarrow F|_{T}^{C}=\bigvee\left\{a \in L \mid T \vdash_{C} E \Rightarrow a \otimes F\right\} .
$$

We now have the following assertion:

Theorem 5 (graded-style completeness). Let $T$ be a C-theory. Then, for any fuzzy attribute $C$ implication $E \Rightarrow F$,

$$
|E \Rightarrow F|_{T}^{C}=\|E \Rightarrow F\|_{T}^{C}
$$

Optionally, we can utilize $\vdash_{C}^{\omega}$ instead of $\vdash_{C}$ in (14) if $\mathbf{L}$ is infinite, see Section 5.2. 


\subsection{Theories as fuzzy sets of formulas}

Graded-style semantic entailment is often considered so that one considers fuzzy sets of formulas as theories. This approach is used in abstract fuzzy logic [14, 17] and was introduced by Pavelka [21]. We now show that both semantic entailment and provability in our logic can be generalized utilizing that concept. Moreover, we show that such an extension is graded-style complete.

Given an L-set $T$ of fuzzy attribute $C$ implications, one can introduce a notion of a model of $T$ as follows. An $\mathbf{L}$-set $M \in \operatorname{fix}(C)$ is a $C$-model of $T$ if $T(E \Rightarrow F) \leq\|E \Rightarrow F\|_{M}$ for all $E, F \in \operatorname{fix}(C)$. Then, a degree $\|E \Rightarrow F\|_{T}^{C}$ to which $E \Rightarrow F$ follows from an $\mathbf{L}$-set $T$ of fuzzy attribute $C$-implications can be defined as in (10) with $\operatorname{Mod}_{C}(T)$ denoting the set of all $C$-models of $T$.

Using a reductionist approach, we can establish a graded-style completeness:

Theorem 6. Let $T$ be an $\mathbf{L}$-set of fuzzy attribute $C$-implications. Then, for any fuzzy attribute $C$ implication $E \Rightarrow F$,

$$
\|E \Rightarrow F\|_{T}^{C}=\bigvee\left\{a \in L \mid T^{\circ} \vdash_{C} E \Rightarrow a \otimes F\right\},
$$

where $T^{\circ}=\{E \Rightarrow T(E \Rightarrow F) \otimes F \mid E, F \in \operatorname{fix}(C)\}$.

Again, $\vdash_{C}$ can be replaced by $\vdash_{C}^{\omega}$ to get a completeness result for arbitrary $\mathbf{L}$.

Remark 9 . The set $T^{\circ}$ is in general infinite and contains redundant formulas. For instance, one can remove from $T^{\circ}$ all formulas $E \Rightarrow T(E \Rightarrow F) \otimes F$ such that $T(E \Rightarrow F) \otimes F \subseteq E$, see [9] for details.

\section{Acknowledgment}

Supported by grants no. P103/10/1056 and no. P103/11/1456 of the Czech Science Foundation and by grant no. MSM 6198959214 .

\section{References}

[1] W. W. Armstrong, "Dependency structures in data base relationships", in: IFIP Congress, Geneva, Switzerland, pp. 580-583, 1974.

[2] M. Baaz: "Infinite-valued Gödel logics with 0-1 projections and relativizations", Lecture Notes in Logic, vol. 6, Springer-Verlag, 23-33, 1996.

[3] R. Belohlavek, Fuzzy Relational Systems: Foundations and Principles, New York: Kluwer, 2002.

[4] R. Belohlavek, T. Funiokova, V. Vychodil, "Fuzzy closure operators with truth stressers", Logic Journal of the IGPL 13, pp. 503-513, 2005.

[5] R. Belohlavek, V. Vychodil, "Fuzzy attribute logic: syntactic entailment and completeness", in: Proc. JCIS 2005, 8th Joint Conference on Information Sciences, Salt Lake City, Utah, USA, pp. 78-81, July 2005.
[6] R. Belohlavek, V. Vychodil, "Fuzzy attribute logic: attribute implications, their validity, entailment, and non-redundant basis", in: Proc. IFSA 2005, vol. I, pp. 622-627, 2005.

[7] R. Belohlavek, V. Vychodil, "Data tables with similarity relations: functional dependencies, complete rules and non-redundant bases", in: Proc. DASFAA 2006, LNCS, 3882, Berlin: Springer-Verlag, pp. 644-658, 2006.

[8] R. Belohlavek, V. Vychodil, "Reducing the size of fuzzy concept lattices by fuzzy closure operators", in: Proc. SCIS \& ISIS 2006, Tokyo Institute of Technology, pp. 309-314, 2006.

[9] R. Belohlavek, V. Vychodil, "Fuzzy attribute logic over complete residuated lattices", $J$. Exp. Theor. Artif. Intelligence, 18, pp. 471-480, 2006.

[10] R. Belohlavek, V. Vychodil, "Query systems in similarity-based databases: logical foundations, expressive power, and completeness", in: Proc. ACM SAC 2016, pp. 1648-1655, 2010.

[11] P. Cordero, M. Enciso, A. Mora, I. P. de Guzmán: A complete logic for fuzzy functional dependencies over t-norms. In: Proc. ESTYLF 2010, pp. 205-210, 2010.

[12] F. Esteva, L. Godo, "Monoidal t-norm based logic: towards a logic for left-continuous tnorms", Fuzzy Sets and Systems 124, pp. 271288, 2001.

[13] B. Ganter, R. Wille, Formal Concept Analysis. Mathematical Foundations, Berlin: Springer, 1999.

[14] G. Gerla, Fuzzy Logic. Mathematical Tools for Approximate Reasoning, Dordrecht: Kluwer, 2001.

[15] J. A. Goguen, "The logic of inexact concepts", Synthese, 18, pp. 325-373, 1968.

[16] R. P. Grimaldi, Discrete and Combinatorial Mathematics, An Applied Introduction, Pearson Education, 2004.

[17] P. Hájek, Metamathematics of Fuzzy Logic, Dordrecht: Kluwer, 1998.

[18] P. Hájek, "On very true", Fuzzy Sets and Systems, 124, pp. 329-333, 2001.

[19] E. P. Klement, R. Mesiar, E. Pap: Triangular Norms, Kluwer, Dordrecht, 2000.

[20] D. Maier, The Theory of Relational Databases, Rockville: Computer Science Press, 1983.

[21] J. Pavelka, "On fuzzy logic I, II, III", Z. Math. Logik Grundlagen Math., 25, pp. 45-52, 119 134, 447-464, 1979.

[22] S. Pollandt, Fuzzy Begriffe, Berlin: SpringerVerlag, 1997.

[23] G. Takeuti, S. Titani, "Globalization of intuitionistic set theory", Annals of Pure and Applied Logic, 33, pp. 195-211, 1987.

[24] W. Wechler, Universal Algebra for Computer Scientists, Berlin: Springer-Verlag, 1992. 